\title{
Oral manifestations of autoinflammatory and autoimmune diseases
}

\author{
Špela Baglama ${ }^{1 凶}$, Katarina Trčko², Janez Rebol ${ }^{3}$, Jovan Miljković ${ }^{1}$
}

\begin{abstract}
Autoimmune diseases may also be reflected in changes in the oral cavity that represent the first sign of the disease, or they may occur simultaneously with or later in the course of the disease. Oral findings are mostly non-specific, and therefore further investigations are needed to exclude or confirm possible diagnoses. This article presents the most important diseases in this research area, divides them into meaningful groups, and highlights the importance of examining the oral cavity for possible manifestations.

Keywords: autoimmune diseases, inflammatory diseases, oral manifestations, mucous membrane, oral medicine

Received: 1 November 2017 | Returned for modification: 10 November 2017 | Accepted: 12 November 2017
\end{abstract}

\section{Introduction}

Various autoimmune diseases may present with heterogeneous lesions in the oral cavity and may involve other extraoral sites as well (1). Moreover, the oral mucosa is frequently the first body area affected in many conditions described later, but oral lesions may also occur simultaneously or as a late event in the course of systemic disease (2). Changes to the oral mucous membrane that have an acute or insidious onset may manifest as blisters and ulcerations, which are the most frequent changes in the oral cavity associated with immune-mediated disorders, erosions, erythema, and last but not least desquamative gingivitis, which describes a clinical condition of erythema, desquamation, and/or erosion on the buccal side of the gingiva. The prevalence of oral cavity involvement varies among different autoimmune diseases, and Table 1 shows those that are known (1). Many autoimmune diseases have similar oral clinical appearances and also resemble oral changes due to other causes; hence differential diagnosis is usually very broad. For accurate and early diagnosis, precise patient history and physical examination of the entire body must sometimes be accompanied by histopathological, immunological, and other laboratory tests $(1,3,4)$. If the localized form is not recognized properly and early enough, the disease can worsen (5). However, a multidisciplinary approach is needed to relieve signs and symptoms in these diseases (2). This review covers the most important autoimmune and inflammatory conditions of the oral cavity, which are divided into suitable groups.

\section{Autoimmune and inflammatory skin diseases}

\section{Pemphigus diseases}

The term pemphigus describes a group of chronic bullous mucocutaneous diseases with autoantibodies against various desmosomal components that result in cell-cell detachment (acantholysis) and consequently in intra-epithelial blister formation $(18,19)$. Pemphigus vulgaris (PV) and paraneoplastic pemphigus (PNP) are the most common diseases in the pemphigus group that may affect the oral mucosa (1). Therefore, this review focuses on them.

\section{Pemphigus vulgaris}

PV is the most common disease in the pemphigus group (3). $\mathrm{Mu}$ cocutaneous manifestations are the result of intercellular autoantibodies mainly of the IgG type working against desmosomal antigens desmoglein 3 (Dsg3) in the mucosal dominant type and additionally against desmoglein 1 (Dsg1) in the mucocutaneous type (20).

The oral cavity is the first body area attacked in $60 \%$ of cases, and lesions are found particularly on the buccal, soft palatal, lingual,

\begin{tabular}{lcc} 
Table 1 | Prevalence of oral cavity involvement in autoimmune and inflammatory diseases. \\
\hline Disease & Prevalence & Reference no. \\
\hline Pemphigus vulgaris & $80-90 \%$ & 6 \\
Mucous membrane pemphigoid & $85 \%$ & 7 \\
Psoriasis & $43 \%$ & 8 \\
Oral lichen planus & $70-77 \%$ & 9 \\
Erythema multiforme & $60-70 \%$ & 10 \\
Stevens-Johnson syndrome and toxic epidermal necrolysis & $71-100 \%$ & 11 \\
Crohn's disease & $0.5-20 \%$ & 12 \\
Recurrent aphthous stomatitis & $100 \%$ & 13 \\
Lupus erythematosus & SLE 8-45\%;CLE 4-25\% & 12 \\
Rheumatoid arthritis & $5-86 \% *$ & 14 \\
Scleroderma & $80 \% \star \star$ & 15 \\
Behçet's disease & $97-100 \%$ & 16 \\
Graft-versus-host disease & $25-80 \%$ & 17 \\
\hline
\end{tabular}

SLE = systemic lupus erythematosus; CLE = cutaneous lupus erythematosus.

* Prevalence of temporomandibular joint disorder.

** Prevalence of orofacial manifestations. 
and labial mucosa $(3,6)$. They start as thin-walled, fluid-filled blisters that easily rupture (and therefore intact blisters are rarely observed), leaving diffuse, painful erosions and ulcerations with a burning sensation, ranging from less than 0.5 to $2 \mathrm{~cm}(1,3,12$, 21). The ulcers are superficial and irregular in shape, their base is red, and their margins are ragged and whitish, but as infection is established a yellowish slough may develop $(1,12)$. Nikolsky sign may be positive (12). The ulcers usually heal in a few weeks without scarring (22). However, given that they are painful, the patient is prevented from feeding sufficiently (21). The gingivae are less frequently affected and changes are usually described as desquamative gingivitis. Lesions on the skin may appear a few months later (1). Other mucosal surfaces may also be affected (3).

Histopathological examination identifies acantholysis, acantholytic cells within intraepithelial vesicles, separated basal keratinocytes, and occasionally eosinophilia $(4,22,23)$. Direct immunofluorescence (DIF) shows intercellular IgG and $\mathrm{C}_{3}$ binding in a netlike pattern. For circulating autoantibodies against epithelial intercellular antigens to be noted, indirect immunofluorescence (IIF) or quantitative immunoassays can be used $(1,22)$ (Fig. 1).

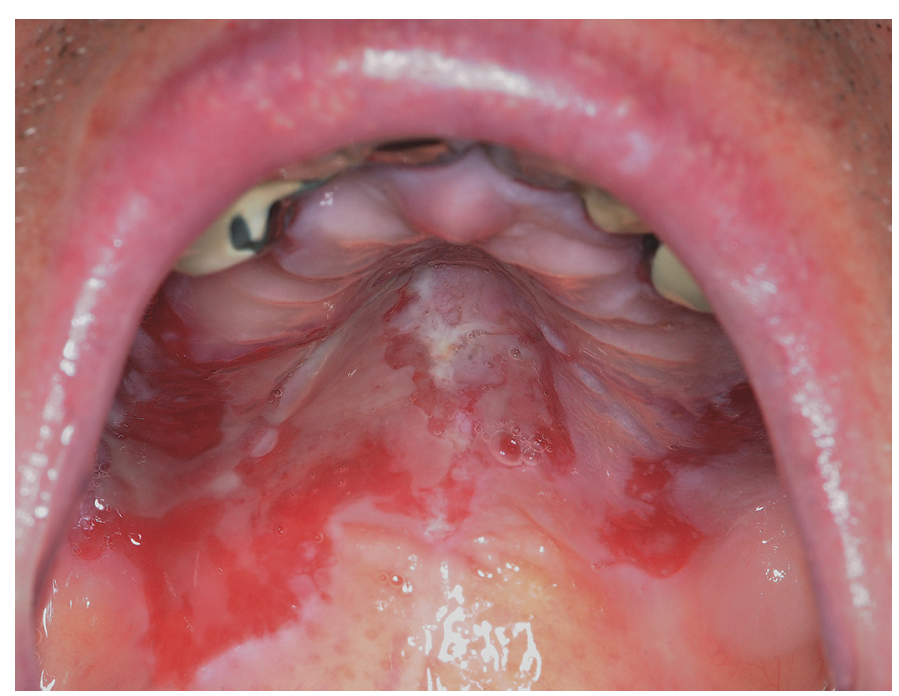

Figure 1 | Pemphigus vulgaris: diffuse erosions and ulcerations involving the hard palate and gingiva.

\section{Paraneoplastic pemphigus}

As the name suggests, PNP is almost always associated with certain neoplastic diseases (1). Most frequently, neoplasm is diagnosed before the development of PNP, but PNP may also be the first indication of underlying malignancy (20). However, autoantibodies against various targets can be found, including desmosomal proteins Dsg1 and Dsg3, plakin family proteins (periplakin, envoplakin, plectin, desmoplakin, and $\mathrm{BP} 180$ ), and the protease inhibitor alpha-2-macroglobulin-like antigen 1 (A2ML1) (3, 20, 23).

Another term for PNP is paraneoplastic autoimmune multiorgan syndrome, which implies that the disease can be manifested by a wide variety of signs and symptoms (20). Changes on the oral mucosa are present in nearly all individuals affected, with the appearance of painful, hemorrhagic erosions at any place in the oral cavity but typically on the lateral part of the tongue and lip vermilion zone $(3,24)$. The next manifestation in the oral cavity is stomatitis; it is noteworthy that this is often among the first indicators of the disease (24). Nikolsky sign may be provoked (25).

Histopathologically, PNP is most often seen as suprabasal acantholysis, dyskeratosis, and cellular autoimmune phenomena: vacuolar interface change and lichenoid infiltrates. DIF re- veals intercellular IgG and/or $\mathrm{C}_{3}$ deposits, sometimes in combination with their linear deposits along the basement membrane (20). IIF detects circulating IgG autoantibodies directed to intercellular surfaces and almost always to the basal membrane zone. Those that are towards plakins are a characteristic feature (24). However, autoantibodies can be further detected by various immunoassays. If underlying malignancy has not yet been established, thorough investigations must be carried out in this direction as well (20).

\section{Pemphigoid diseases}

Pemphigoid diseases are a heterogeneous group of chronic autoimmune disorders that are characterized by autoantibodies directed against various structural proteins of the hemidesmosomes, resulting in epithelial detachment (18). This article covers mucous membrane pemphigoid (MMP) and epidermolysis bullosa acquisita (EBA).

\section{Mucous membrane pemphigoid}

MMP, also known as cicatricial pemphigoid, most commonly involves the mucosal surfaces and hardly ever the skin (7). Autoantibodies, predominantly of IgG type, to several antigens within the basement membrane zone have been noted, including $\mathrm{BP} 230$, BP180, $a-6$ and $\beta-4$ integrin subunits, laminin-5, laminin-6, collagen type VII, and $120-\mathrm{kDa}$ undefined epithelial antigen, suggesting that mucous membrane pemphigoid is a heterogeneous group of several subtypes of the disease $(23,26)$.

Oral manifestations normally cover the palatal, gingival, labial, tongue, and buccal mucosa. They can be described as erythema, erosions covered with a pseudomembrane, ulcers, and at times intact vesicles or bullae (7). However, the gingiva is the site most often affected, frequently by so-called desquamative gingivitis $(7,20)$. Nikolsky sign may be positive (23). Lesions in the oral cavity usually heal in 7 to 10 days and seldom scar regardless of the tendency to scar elsewhere $(22,23)$. Patient issues can include pain, bleeding, dysphagia, and loss of gum with subsequent tooth loss $(3,23)$. Furthermore, the possible risk of developing various solid tumors in anti-laminin 5 MMP cases must be emphasized (1). Despite this, individuals with only oral cavity involvement have a very good prognosis (26).

Under light microscopy, epithelium detachment from underlying inflamed connective tissue, more precisely at the level of the lamina lucida, is visible $(20,22)$. Chronic inflammatory infiltrate mostly consists of lymphocytes and to a lesser extent eosinophils and neutrophils $(1,7)$. DIF findings are $\mathrm{C}_{3}$, IgG, and/or IgA deposits

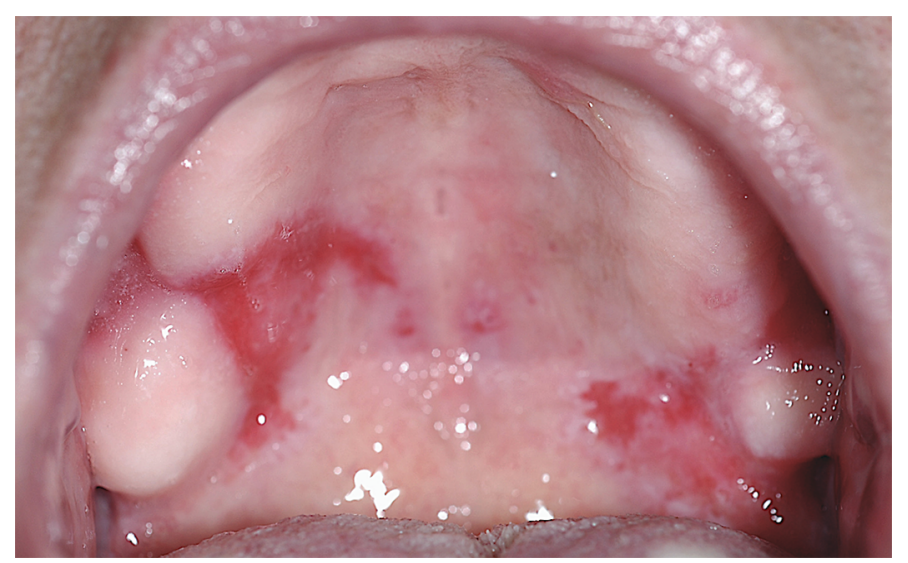

Figure 2 | Mucous membrane pemphigoid: erosions on palatal and gingival mucosa. 
in the linear pattern along the basement membrane zone $(20,26)$. Salt-split IIF may also be performed to differentiate MMP from EBA (23). Diagnostic dilemmas may be elucidated with more advanced immunologic methods (7). The above mentioned subgroup of MMP should be properly monitored for possible neoplasms (26) (Fig. 2).

\section{Epidermolysis bullosa acquisita}

EBA is an acquired, chronic, bullous mucocutaneous condition in which autoantibodies mainly of IgG type against type VII collagen in the basement membrane are present $(1,3,23)$. Separation of the epidermis from the dermis follows (27).

A key feature is that even mild mechanical trauma leads to the emergence of clinical changes (23). Mucosal surfaces are affected in $60 \%$ of cases and they are a predominant disease area in the mucous membrane pemphigoid-like variant, which is one of the five subtypes of the disease (27). The appearance of oral lesions may include blisters, erosions, tooth loss, and mandibular contraction with consequently limited extent of mouth opening (3). They are prone to healing with scarring. Dental treatment should be avoided as far as possible or a bolus of high-dose corticosteroids should be applied immediately prior to it (23).

To make a diagnosis, histology, DIF, and serological analysis are crucial $(1,3)$. A histological sample reveals subepidermal blisters with various inflammatory cells in them. DIF shows linear deposits of IgG and/or IgA autoantibodies at the basement membrane zone in a u-serrated pattern. IIF or enzyme-linked immunosorbent assay (ELISA) detects circulating autoantibodies against type VII collagen $(1,27)$.

\section{Psoriasis}

Psoriasis is a chronic, multifactorial skin disease that is characterized by autoimmune and autoinflammatory processes, complex genetics, dysregulation of nearly all skin cells, various biochemical abnormalities, and abnormal papillary capillaries $(28,29)$.

The actual existence of oral psoriasis is still questionable despite all of the published studies. It is assumed to be rare and is also rarely recognized (30). So far, in the literature very diversely described oral psoriatic patterns have been recorded, including white, erythematous, and mixed ones-each with different more explicit features (31). The buccal mucosa is the most common site. Lesions are predominantly asymptomatic and transient (30). Some studies suggest the possibility of more frequent geographic tongue and fissured tongue development in psoriatic patients, but their simultaneous presence may simply be coincidental (31). Frequent occurrence of gingivitis is yet another finding observed in the literature. Psoriatic involvement of the lips and perioral region is also mentioned, but the clinical appearance is very diverse and therefore can be misidentified for other possible diagnoses. Applying to psoriatic arthritis, it is important to mention possible problems with oral hygiene. However, the links between periodontal disease and psoriasis need to be fully established. It is believed that most often oral manifestations appear in relation to generalized pustular or erythrodermic psoriasis (30).

It should be noted that at present any lesion in the oral cavity is difficult to unambiguously associate with psoriasis, owing to the fact that the relevant clinical and histopathological criteria are still not available along with the existence of many differential diagnostic possibilities $(30,31)$. Potential irritants must be removed because they permit new changes to appear (30) (Fig. 3).

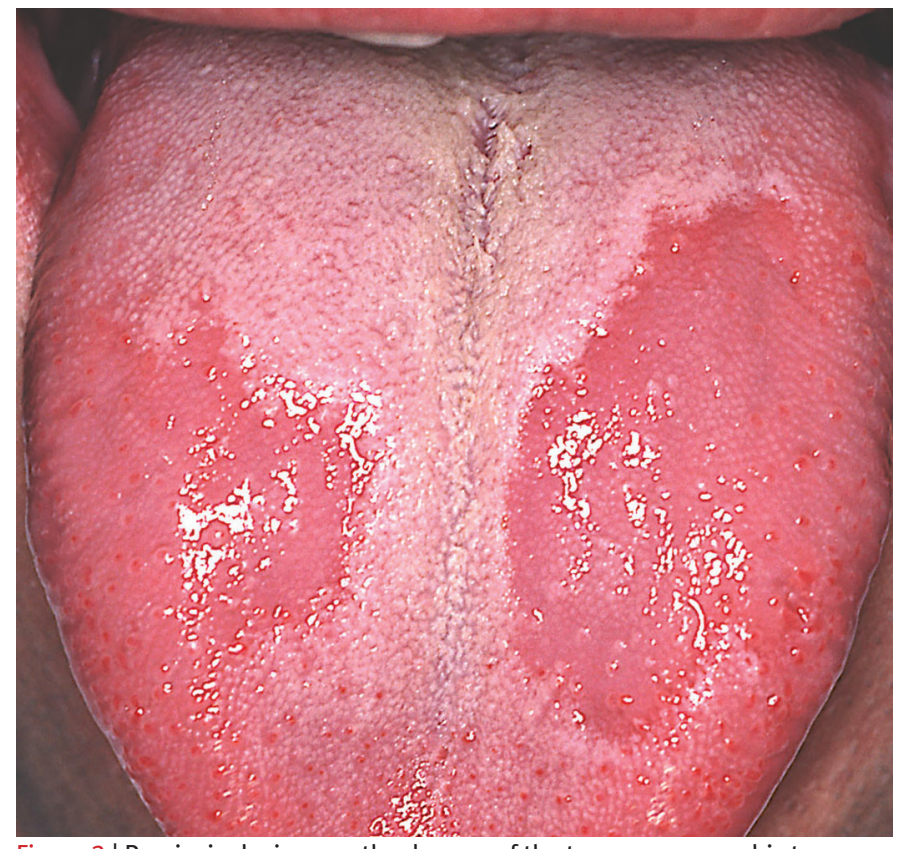

Figure 3 | Psoriasis: lesions on the dorsum of the tongue, geographic tongue.

\section{Oral lichen planus}

Lichen planus (LP) is an inflammatory disease that most frequently affects the oral mucosa and skin, but can also involve other mucosal surfaces, the nails, and the hair. This review focuses on oral LP (OLP), which is a subtype of LP with a typical chronic course of relapses and remissions (9). The pathogenesis has yet to be further explored. However, it is believed that T-cell-mediated damage to the basal keratinocytes and consequently apoptosis activation is the basis of the disease $(3,23)$. A few characteristics could indicate an autoimmune nature of the disease (1).

The sites in the oral cavity most often affected are the tongue, buccal area, labial mucosa, and gingiva, where desquamative gingivitis is usually found (32). The Koebner phenomenon is a wellknown precipitating factor (3). However, oral manifestations can be further divided into six subgroups: reticular, papular, plaque, atrophic, erosive, and bullous. Reticular OLP is the most common, and its appearance consists of white, flat papules with a distribution on the mucosa typically creating a lace-like pattern (5). Wickham striae are also present (1). In the papular type, white papules measure 0.5 to $1.5 \mathrm{~mm}$ in diameter. The plaque type manifests as white homogenous patches, hence it is similar to leukoplakia (5). In atrophic OLP, areas of erythema and atrophy can be recognized. Finally, the last two subgroups appear as erosions and blisters leaving ulcers, respectively (1). Lesions in the oral cavity are most commonly multiple with a bilateral, symmetrical distribution, and different subgroups can be simultaneously present in the same person (23). Patients are usually asymptomatic; on the other hand, burning, itching, taste disturbances, or very rarely pain due to ulcers can be described $(23,32)$. In addition, xerostomia may also be present, the causes of which can be debated (33). It is important to be aware of the possible progression of mucous changes into squamous cell carcinoma, with the highest recorded frequency in atrophic, erosive, and bullous $\operatorname{OLP}(1,3,32)$. For this reason, regular monitoring for oral cancer is indicated (9). Finally, in some patients it is possible for oral changes to heal with scarring (23).

The diagnosis can be made only on the basis of a characteristic clinical picture (23). The history should also cover a review of systems (32). To simplify matters, a description of the histopatho- 
logical sample is a lichenoid reaction pattern consisting of subepithelial lymphocytes and liquefaction degeneration of basal keratinocytes $(1,4,20,21,32)$. The typical pattern shown on the DIF is linear fibrinogen deposition along the basement membrane zone and often Civatte bodies with positive IgM (20). IIF is negative (23). In cases of uncertainty, skin patch testing can be performed to identify any allergy to dental material to distinguish OLP from oral lichenoid contact lesions (32). Likewise, a precise history of drugs with subsequent withdrawal of the potential agent can be carried out if a lichenoid drug reaction is likely to be the cause (9) (Fig. 4).

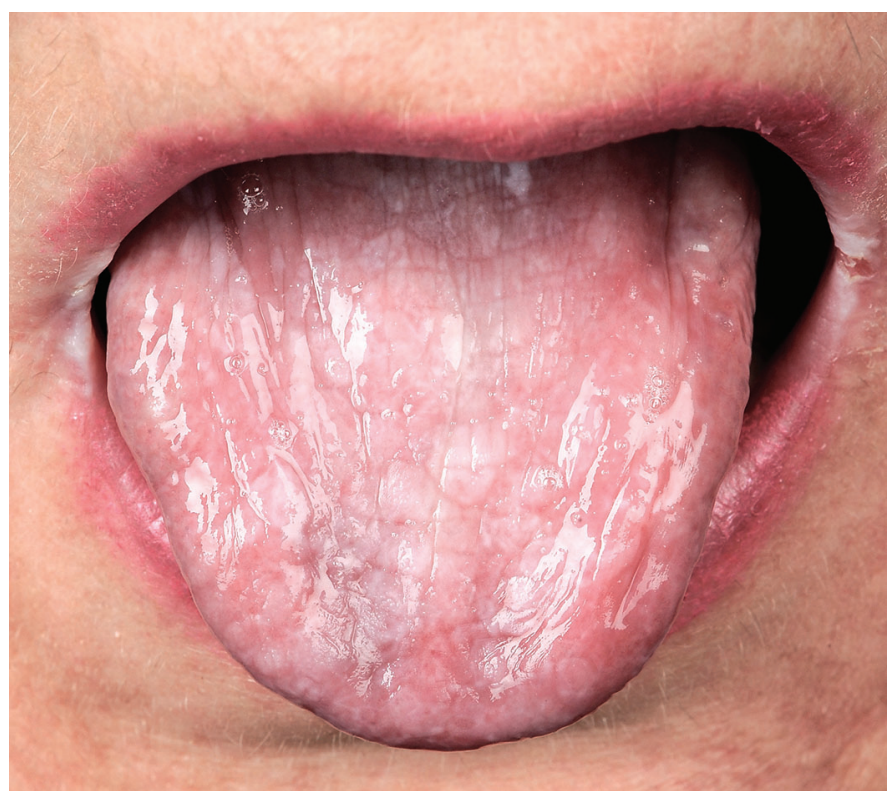

Figure 4 | Oral lichen planus: atrophic areas and white patches on the tongue.

\section{Erythema multiforme, Stevens-Johnson syndrome, and toxic epidermal necrolysis}

Erythema multiforme (EM) is an acute mucocutaneous disease with classic target-like skin lesions that has been postulated as a type IV hypersensitivity reaction to various causative factors, with herpes simplex virus being the most common $(3,10)$. Cytotoxic intra- and subepithelial damage follows, possibly due to non-self antigens on keratinocytes. In addition, it has been proven that some patients have autoantibodies attacking the epidermis, and desmoplakin 1 and 2 (34). EM can be subdivided into EM major and minor, with differences in mucosal involvement and clinical course $(1,22)$. Although EM is largely self-limiting within a few weeks, a recurrent form of the disease also exists, with documented association with herpes simplex virus between 61 and 100\% (10).

However, the mucosal surfaces in EM are only slightly affected; in fact, among them the oral cavity most commonly undergoes changes $(3,23)$. Broadly speaking, erythema and vesicles leaving painful pseudomembrane-covered ulcerations or necrotic mucosa can be found, particularly on the buccal and labial mucosa as well as the vermilion border $(1,3,10,21,23)$. In addition, bloodstained crusts on the lips with subsequent speech and feeding problems are another notable manifestation $(1,3,23)$. In the case of changes affecting only the oral cavity, the term oral EM is used (1). This form is recurrent with repetition from every 3 weeks to once a year and lasts from 10 days to 6 weeks (34).

In making a diagnosis, the abrupt onset of the disease, causative factors in patient history, and typical target-like lesions are very important indicators $(1,3)$. Histology reveals non-specific inflammation with prominent epithelial necrosis. DIF and IIF are also non-specific. To help, laboratory tests may be carried out to confirm or exclude recent infections, principally herpes simplex virus 1 and 2 as well as Mycoplasma pneumoniae (23). A cutaneous patch test can also be helpful in detecting the causative factors (34).

Stevens-Johnson syndrome (SJS) and toxic epidermal necrolysis (TEN) are entirely distinct groups of disorders sharing many similarities with each other (3). Research suggests that genetic and innate immune mechanisms are combined in pathogenesis, with subsequent activation of apoptosis and secondary epidermal necrosis, which ultimately leads to the death of keratinocytes (35). In these multiorgan disorders not only is mortality high, but survivors may also have long-term complications. However, TEN is more serious than SJS (3). Children are more likely to have a repetitious course (35).

The coverage of mucosal and skin surfaces is extensive; specifically, at least two mucosal areas must be involved $(11,23)$. In the oral cavity, blisters leaving ulcers can be expressed. Notably, crusted lips are a typical discovery. The gingiva is rarely affected (36). Nikolsky sign is positive (35). In addition, long-term sequelae in the mouth are possible, including scarring, synechiae, persistent tongue papillae loss, anomalous roots, hypoplasia and growth abnormalities of permanent teeth, increased occurrence of caries and candidiasis, and finally less saliva production with changed quality (11).

The history and clinical appearance are the most important for making a diagnosis. The histology is non-specific (36).

\section{Autoimmune and inflammatory diseases of the digestive tract}

\section{Crohn's disease}

Inflammatory bowel disease is a chronic inflammatory condition that consists of two main subsets; namely, Crohn's disease (CD) and ulcerative colitis. This review discusses CD (37). CD is a multifactorial condition with still insufficiently clear connections between genetic susceptibility, autoimmunity, and environmental triggers (2). It can be manifested in any segment of the digestive tract from the oral cavity to the anus, as well as almost anywhere outside it (38).

However, despite various studies, it is not yet clear why oral lesions occur only in some patients, although it seems that extraintestinal manifestations occur because of sharing common epitopes with inflamed intestinal mucosa $(2,38)$. Oral changes can precede intestinal involvement or occur simultaneously, but they generally appear after intestinal involvement $(37,38)$. Patients can develop changes specific to $\mathrm{CD}$; for example, painless swollen lips, fissuring of the tongue and lips, cobblestone appearance of hyperplastic mucosa, deep linear ulcers mainly in the buccal vestibule, mucogingivitis, and, due to fibrosis, mucosal tags, nodules, or polyps $(2,12,38)$. Although these alterations are characteristic of $\mathrm{CD}$, they do not coincide with its dynamics (37). It should be emphasized that non-specific changes due to complications caused by nutritional deficiencies, side effects of prescribed drugs, and chronic inflammation are also possible (38). Among other things, in the group of non-specific lesions there are aphthous ulcers, angular cheilitis, pyostomatitis vegetans, persistent submandibular lymphadenopathy, lichenoid reactions, EM, SJS, glossitis, depapillation of the tongue, halitosis, dental erosion and caries, periodontal disease, spontaneous bleeding, oppor- 
tunistic infections, mucosal atrophy, perioral erythema, pale mucosa, gingival hyperplasia, stomatitis, erosions, and ulcerations, whereas symptoms sometimes described include odynophagia, dysphagia, tongue pain, taste disturbances, and a burning sensation $(37,38)$. Moreover, xerostomia often occurs secondary to the involvement of minor salivary glands, or chronic inflammation may cause obstruction of the salivary ducts with subsequent consequences (38). It is assumed that all of these manifestations are more common among children and adult males, and that nonspecific ones predominate over specific ones. It should not be forgotten that chronic inflammation and the therapy used can give rise to malignant diseases of the oral cavity $(2,38)$.

Histologically non-caseous granulomas can typically be visible in specific lesions along with chronic inflammatory infiltrate (38, 39). To confirm the disease, radiographic examination, endoscopy, and histopathology are the standard approach. In aid, basic laboratory tests and additional antibody studies may be carried out (40).

\section{Recurrent aphthous stomatitis}

Recurrent aphthous stomatitis (RAS) is the most frequent inflammatory ulcerous disorder of the oral cavity and it repeats in periods from a few days to a few months with a generally rarer occurrence over the years $(41,42)$. The role of various trigger factors is assumed. As far as pathogenesis is concerned, there is not yet a fully completed theory of imbalance in pro- and anti-inflammatory cytokines with resultant autoimmunization in predisposed individuals (41).

First, 2 to 48 hours before ulcers appear, prodromal burning or tingling occasionally occurs together with localized erythema. One or more circular painful ulcers that have a clear-cut erythematous rim and shallow center covered with a yellowish-gray pseudomembrane follow $(13,42)$. These ulcers are divided into minor, major, and herpetiform. Features of minor RAS, which covers more than $85 \%$ of all cases, are diameter up to $1 \mathrm{~cm}$ and non-scarring healing in 7 to 10 days $(13,21,42)$. Major aphthae are larger in diameter, continue for weeks to months, and eventually scar. The later form is also called Sutton's disease or periadenitis mucosa necrotica recurrens. Last, and most rarely, the herpetiform form exists with numerous 2 to $3 \mathrm{~mm}$ ulcers distributed all over the mucosa. They can fuse into wide ulcerous areas (42). However, characteristic oral mucosal locations of the RAS are the buccal and labial mucosa as well as tongue (41).

Typically, RAS is diagnosed on the basis of extensive patient history, including a review of systems, and clinical examination $(2,42)$. In the case of persistence of ulcers for more than 2 or 3 weeks, laboratory tests and then a biopsy should be performed (2). It should be noted that, if RAS first appears during adulthood or exacerbates at that time, then the possibility of an underlying systemic cause must be considered (13).

\section{Systemic autoimmune and inflammatory diseases}

\section{Sjögren's syndrome}

Sjögren's syndrome (SS) is a chronic autoimmune disease causing lymphocytic infiltration of the exocrine glands, especially the salivary and lacrimal ones (43). In addition, it is characterized by various autoantibodies such as anti-Ro and anti-La (44). Attributes connected to SS are B-cell hyperactivity leading to accumula- tion of autoantibodies, hypergammaglobulinemia, and the development of ectopic lymphoid foci in inflamed tissue (3). Two types of the disease are known: primary and secondary SS, based on the linkage with other autoimmune diseases (14).

In $80 \%$ of cases there is an insidious onset of sicca symptoms. Mostly, keratoconjunctivitis sicca is the first and xerostomia is the next in the sequence of various manifestations. Rarely, dry mouth is present from the very beginning and other symptoms appear later or different sicca symptoms develop concurrently. However, changes on any part of the body can be observed (43). As a consequence of quantitative and qualitative alterations in the saliva, the complaints described in the next few sentences are found in the oral cavity (14). Colonies of Lactobacillus acidophilus, Streptococcus mutans, and Candida albicans are multiplied, regardless of unaltered total salivary microbial count. Therefore, dental plaques are more cariogenic and acidophilic. This may be a reason for the higher prevalence of caries and candidiasis among affected subjects (44). Candidiasis usually appears as angular cheilitis, atrophy, or erythema of the mucosa (45). Some studies also indicate an elevated prevalence of periodontal disease; however, this does not apply to all of them (44). Other possible unfavorable oral complications are erosions, ulcers, mucosal friability, cracked lips, depapillation of the tongue, halitosis, mucositis, and swelling of the salivary glands. Patients often complain about dryness of the mouth and impaired tasting, swallowing, and speaking; furthermore, they may also have problems with coughing, hoarseness, and dyspepsia, along with other symptoms (14, 45, 46). The worst complication of SS is non-Hodgkin lymphoma, with a 20 -fold increased risk (45).

The diagnosis of SS can be made on the basis of the American European Consensus Group criteria (46). Serology is meaningful when it shows positive anti-Ro/La autoantibodies or rheumatoid factor plus ANA (any) $(43,45)$. In any case of xerostomia, mycological and bacteriological samples should be taken (46).

\section{Lupus erythematosus}

Lupus erythematosus (LE) is a chronic multisystem condition with an autoimmune background. It can be divided into two categories: systemic LE (SLE) and cutaneous LE. The most characteristic autoantibodies involved in the pathogenesis are antinuclear ones; specifically, in SLE anti-ds-DNA and anti-Sm (1).

For some individuals, abnormalities in the oral cavity may represent the only change. However, certain alleles of STAT4 have been shown to be protective against ulcer formation in lupus. There are a wide variety of possible changes in the oral mucosa; hence it is difficult to describe all of them. The typical variation is represented as erythema or erosion together with white papules in its center and white keratotic striae in the periphery, sometimes with telangiectasia. Among other nonspecific lesions are honeycomb plaques (white reticular plaques), lifted keratotic plaques, nonspecific erythemas, purpura, petechiae, and ulcers $(12,47)$. Discoid lesions on the lip vermilion and cheilitis can also be found; of note, these lesions spread into perioral skin $(3,12)$. Then, secondary SS and associated problems can simultaneously be present in up to $30 \%$ of SLE patients (5). Moreover, secondary infections may occur, particularly viral and fungal in the oral cavity (2). There are several possible reasons for this (47). Oral manifestations are usually multiple in asymmetric arrangement, generally found on the lips, buccal mucosa, hard palate, and gingiva. They may be asymptomatic in up to $50 \%$ of cases (1). However, 
patients may complain about xerostomia, stomatodynia, and dysgeusia (5). Importantly, the occurrence of oral lesions can be an indicator of increased disease activity (2). The possibility of malignant transformation into squamous cell carcinoma in the case of persistent ulcers or a hard lump must be excluded (47).

Histopathological findings are hyperkeratosis, liquefaction of the basal cell layer, thickening of the basement membrane, and interface mucositis with lymphocytic inflammatory infiltrate distributed diffusely, deep, and perivascular in the connective tissue $(1,48)$. DIF features are linear deposition of IgG or IgM and/or $\mathrm{C}_{3}$ in the basement membrane (2). Serological tests should also be performed (48) (Fig. 5).

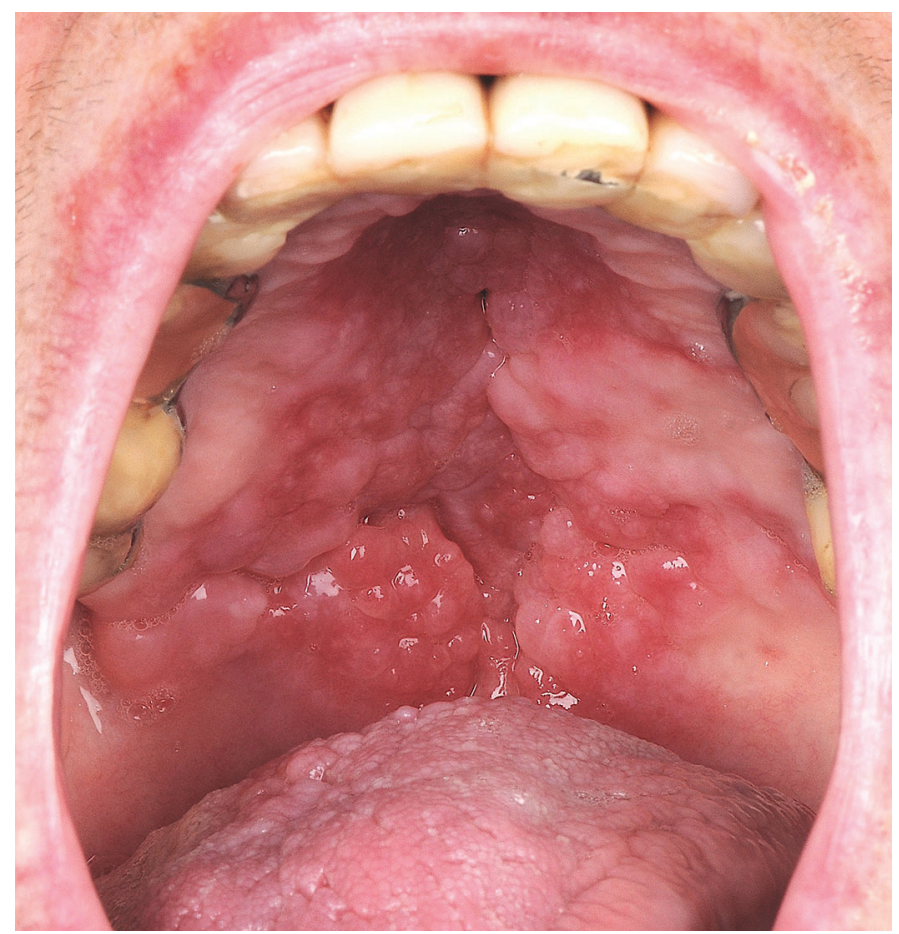

Figure 5 | Lupus erythematosus: multiple papules covering erythematous palatal mucosa.

\section{Rheumatoid arthritis}

Rheumatoid arthritis (RA) is a chronic autoimmune inflammatory disease with bilateral symmetric polyarthritis as its most common feature. In addition, other tissues may be damaged. The pathogenesis most likely involves the intertwining of genotype and environmental causative factors, among them the periodontal microbe Porphyromonas gingivalis (2).

In the oral cavity, RA can be related to temporomandibular joint disorder (TMJD), which is the most common orofacial manifestation, secondary SS, and perhaps periodontal disease $(14,49)$. The connection between periodontal disease and RA is not yet fully explained, but some studies have demonstrated similarities in the pathogenesis and clinical features of both $(14,15)$. However, this elevated periodontal risk may be linked to increased dental plaque, which may occur as a result of difficulty in brushing the teeth due to disease-related changes in the joints. On the other hand, both of these can be a consequence of a reduced quantity of salivary secretion in patients with RA (49). Next, TMJD is a non-specific issue that can appear as severe bilateral pain in various areas of the head, triggered by activity. Among the clinical signs are malocclusion, limited oral opening, joint stiffness in the morning, inflammation of the pre-auricular region, and intracapsular crepitus. It occurs more often in children with RA than in adults, possibly leading to mandibular growth restriction with subsequent micrognathia and/or ankylosis (14). Finally, secondary SS can be found in up to one-third of sufferers with RA (2).

Patient history, clinical examination, and imaging techniques are tools for making a diagnosis $(2,14)$.

\section{Polymyositis and dermatomyositis}

Polymyositis (PM) and dermatomyositis (DM), subsets of idiopathic inflammatory myopathies (IIM), are autoimmune multifactorial diseases of muscle and other tissues $(50,51)$. More precisely, PM is a systemic disease of the connective tissue (14). On the other hand, it is assumed that DM is a systemic complement-mediated microvasculopathy (51).

In PM, impairment of the pharyngolaryngeal muscles can cause dysphonia and dysphagia, with aspiration and hypersalivation as the end results. In case reports, ulcerations of the tongue and its atrophy have also been demonstrated (14). Similar to PM, DM may lead to oropharyngeal muscle involvement with all of the issues that follow. Dysphagia is associated with aspiration pneumonia and indicates a worse prognosis (52). In addition, if the tongue is affected, this causes macroglossia and its hypotonia with even greater difficulties in speaking, swallowing, and chewing (14). Some case reports describe the occurrence of squamous cell carcinoma of the tongue (52). In addition, on the oral mucosa, ulcers, white lesions, edema, erythema, prominent blood vessels, and gingival telangiectasia can be found. Patients can also have xerostomia and salivary hypofunction $(14,52)$. Furthermore, one study discovered limited mouth opening. It should also be mentioned that muscle weakness can lead to inability to maintain satisfactory oral hygiene, with consequent caries and periodontal disease. Interestingly, oral manifestations are sometimes the first sign in the course of the disease (52).

Diagnostic criteria are helpful in setting up a diagnosis of IIM. Extended laboratory examinations are first performed. Recommended serological tests include the detection of muscle enzymes, especially creatinine kinase, and autoantibodies: myositis-specific and myositis-associated (50-52). If there is a suspicion of malignancy, the risk of which is higher in DM compared to PM, appropriate investigations must be carried out. However, these two diseases can be separated from one another according to typical skin changes in DM in addition to histopathological findings in the muscle biopsy and autoantibody profile. More thorough differences among them will be shown by the International Myositis Classification Criteria Project classification criteria (50).

\section{Scleroderma}

Scleroderma is an autoimmune fibrosing disease that has two major forms: localized scleroderma/morphea and systemic sclerosis (53). The pathogenesis is believed to depend on endothelial cell damage and fibroblast activation with subsequent excessive collagen as well as extracellular matrix component production (15). However, there are some pathogenic dissimilarities between the main forms (53).

Oral manifestations are often among the first clinical signs of the disease (54). Secondary to fibrosis, microstomia can occur (14). Fibrosis therefore leads to speech and swallowing difficulties (54). Moreover, the tongue may become rigid and the gingiva firm in addition to turning pale (5). Case reports have mentioned Raynaud's phenomenon on the tongue (15). Tongue cancer is also 
possible (14). Many patients are also reported to have xerostomia and part of them meet the criteria for SS (15). One recent study found a common occurrence of dysphagia, which can happen due to various causes (54). Furthermore, radiological jaw changes may be observed, but limited mouth opening is more likely the end result of facial tissue fibrosis $(15,54)$. In addition, more pronounced dental caries and periodontal disease are also associated with the disease. The main reasons are most likely difficulty brushing the teeth and xerostomia $(14,54)$. Finally, telangiectasias can be observed on mucous membranes (54).

In addition to clinical findings, serological tests for detecting autoantibodies, specifically antinuclear ones, are important (53).

\section{Behçet's disease}

Behçet's disease is known as a chronic multisystem inflammatory relapsing and remitting condition $(2,21)$. The pathogenesis is still quite obscure. However, some evidence in the research suggests an autoinflammatory background, whereas others contest this (16).

The mouth is commonly affected and lesions can appear as painful ulcerations, which are much the same as in RAS, most often like its major form $(5,13)$. Most frequently they can be found on the labial, buccal, and lateral tongue mucosa (16). It is noteworthy that they are generally the first and most common manifestation of the disease, followed by systemic issues $(2,15)$. As a rule, aphthae must recur at least three times a year (16).

The point system of the International Criteria for Behçet's Disease can be used to determine a diagnosis that relies on clinical features (2, 21) (Fig. 6).

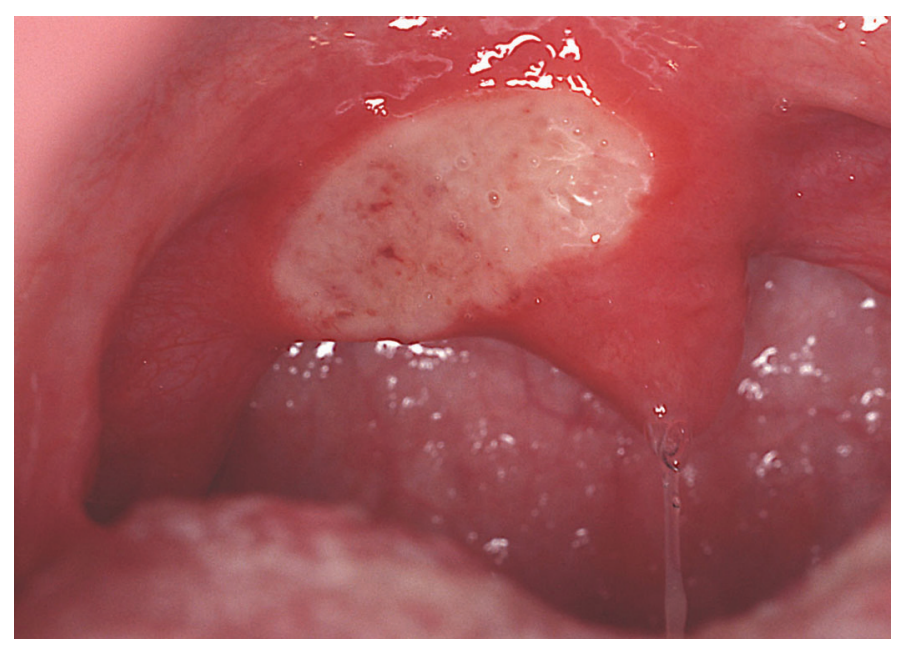

Figure 6 | Behçet’s disease: aphthous ulceration covering the soft palate.

\section{Graft-versus-host disease}

Graft-versus-host disease (GVHD) is a systemic autoimmune and alloimmune disease that is one of the most common and troublesome adverse events of allogeneic hematopoietic stem cell transplant. After transplantation, it may happen that, due to various risk factors, donor T-cells attack antigens on the recipient cells. The resulting immune reaction leads to impairment of various tissues (55). Perhaps autoimmunity is also involved (2). Based on clinical presentation, two categories of the disease are known: acute and chronic (55). The next two paragraphs of this article focus on oral chronic GVHD.

Prevalent locations in the mouth are the buccal mucosa and the tongue (56). Some literature divides oral manifestations into three groups, and one recent study found no association among them, which indicates that oral chronic GVHD consists of three completely separate diseases: mucosal lesions, salivary gland dysfunction, and mouth sclerosis. First of all, mucosal changes can be described as erythema, lichenoid lesions, atrophy, hyperkeratosis, ulcers, mucoceles, pseudomembrane, edema, and cellulitis (17). The disease subsequently often affects the salivary glands, with consequently similar complications as mentioned in SS. Finally, sclerotic changes can be seen, followed not only by limited mouth opening and tongue movement, but also dysphagia. It should also be mentioned that infections, especially candidiasis, are common secondary manifestations with various causative factors (56). Affected individuals typically report worse symptoms than clinicians would expect based on the clinical signs. However, oral features do not affect patient survival, but may still exist after changes in other tissues subside $(55,56)$. A potential oral squamous cell carcinoma occurrence has also been reported; hence patients should be repeatedly monitored (2).

An oral examination should consist of oral mucosal inspection, a 5-minute saliva test, and maximum mouth-opening measurement (17). In the case of exclusive presence of oral manifestations without the involvement of other organs, an oral biopsy sample might have to be taken to establish a diagnosis. Histologically, dyskeratotic epithelial cells, apoptosis, and an inflammatory infiltrate of lichenoid appearance under the basement membrane, consisting of $\mathrm{CD}_{3}+$ and $\mathrm{CD} 68+\mathrm{T}$ cells, can be seen. Moreover, fibrosis due to collagen deposits and atrophy are hallmarks of chronic GVHD (55). In addition, swabs must also be taken to exclude infections (2) (Fig. 7).

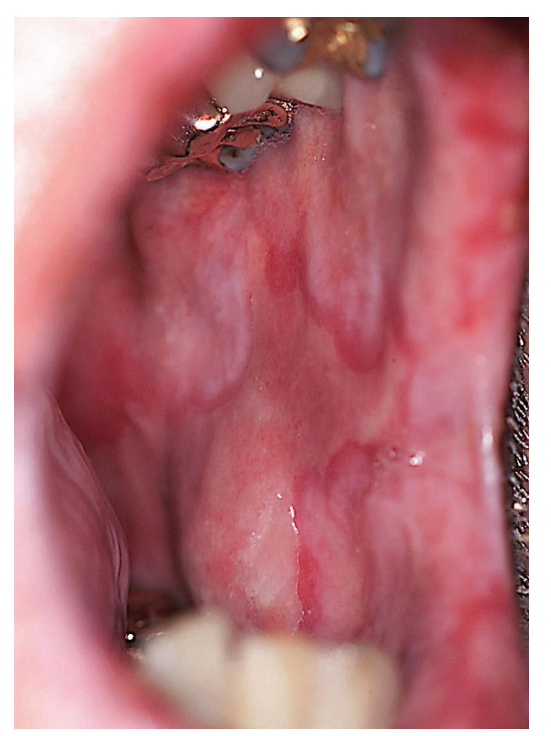

Figure 7 | Oral chronic graft-versus-host disease: erythematous areas, lichenoid lesions, and atrophy on the buccal mucosa.

\section{Conclusion}

As can be seen from this article, oral signs and symptoms may accompany numerous autoimmune diseases. The manifestations are very similar, and yet varied, especially if clinical changes in the entire body are taken into account. Only a handful of all possible diseases are described, and certain detailed information has only been mentioned in passing. In order for readers to gain a more comprehensive perspective, they must read literature focusing on individual phenomena. 


\section{References}

1. Mustafa MB, Porter SR, Smoller BR, Sitaru C. Oral mucosal manifestations of autoimmune skin diseases. Autoimmun Rev. 2015;14:930-51.

2. Mays JW, Sarmadi M, Moutsopoulos NM. Oral manifestations of systemic autoimmune and inflammatory diseases: diagnosis and clinical management. J Evid Base Dent Pract. 2012;12:265-82.

3. Bascones-Martinez A, Garcia-Garcia V, Meurman JH, Requena-Caballero L. Immune-mediated diseases: what can be found in the oral cavity? Int J Dermatol. 2015;54:258-70.

4. Rameshkumar A, Varghese AK, Dineshkumar T, Ahmed S, Venkatramani J, Sugirtharaj G. Oral mucocutaneous lesions-a comparative clinicopathological and immunofluorescence study. J Int Oral Health. 2015;7:59-63.

5. Sridevi P, Munisekhar MS, Harika CH, Rama Krishna A. Oral manifestations of autoimmune diseases. Int J Oral Maxillofac Pathol. 2012;3:27-33.

6. Arpita R, Monica A, Venkatesh N, Atul S, Varun M. Oral pemphigus vulgaris: case report. Ethiop J Health Sci. 2015;25:367-72.

7. Xu H-H, Werth VP, Parisi E, Sollecito TP. Mucous membrane pemphigoid. Dent Clin North Am. 2013;57:611-30.

8. Darwazeh AM, Al-Aboosi MM, Bedair AA. Prevalence of oral mucosal lesions in psoriatic patients: a controlled study. J Clin Exp Dent. 2012;4:e286-91.

9. Alrashdan MS, Cirillo N, McCullough M. Oral lichen planus: a literature review and update. Arch Dermatol Res. 2016;308:539-51.

10. Celentano A, Tovaru S, Yap T, Adamo D, Aria M, Mignogna MD. Oral erythema multiforme: trends and clinical findings of a large retrospective European case series. Oral Surg Oral Med Oral Pathol Oral Radiol. 2015;120:707-16.

11. Saeed H, Mantagos IS, Chodosh J. Complications of Stevens-Johnson syndrome beyond the eye and skin. Burns. 2016;42:20-7.

12. Chi AC, Neville BW, Krayer JW, Gonsalves WC. Oral manifestations of systemic disease. Am Fam Physician. 2010;82:1381-8.

13. Akintoye SO, Greenberg MS. Recurrent aphthous stomatitis. Dent Clin North Am. 2014;58:281-97.

14. Abrao AL, Santana CM, Bezerra AC, Amorim RF, Silva MB, Mota LM, et al. What rheumatologists should know about orofacial manifestations of autoimmune rheumatic diseases. Rev Bras Reumatol. 2016;56:441-50.

15. Hamburger J. Orofacial manifestations in patients with inflammatory rheumatic diseases. Best Pract Res Clin Rheumatol. 2016;30:826-50.

16. Mat MC, Sevim A, Fresko I, Tüzün Y. Behçet's disease as a systemic disease. Clin Dermatol. 2014;32:435-42.

17. Bassim CW, Fassil H, Mays JW, Edwards D, Baird K, Steinberg SM, et al. Oral disease profiles in chronic graft versus host disease. J Dent Res. 2015;94:547-54.

18. Burgdorf W, Plewig G, Wolff HH, Landthaler M, editors. Braun-Falco's Dermatology, 3rd ed. Berlin: Springer-Verlag; 2009. Chapter 46, Autoimmune bullous diseases; p. 641-66.

19. Bolognia JL, Jorizzo JL, Schaffer JV, editors. Dermatology: 2-Volume Set, 3rd ed. Philadelphia: Elsevier Saunders; 2012. Chapter 29, Pemphigus; p. 461-75.

20. Magliocca KR, Fitzpatrick SG. Autoimmune disease manifestations in the oral cavity. Surg Pathol Clin. 2017;10:57-88.

21. Siu A, Landon K, Ramos DM. Differential diagnosis and management of oral ulcers. Semin Cutan Med Surg. 2015;34:171-7.

22. Mayank M, Shah AA, Koshy AV, Mulla A. Immune mediated disorders of oral cavity. Univ Res J Dent. 2017;5:54-8.

23. Schifter M, Yeoh SC, Coleman H, Georgiou A. Oral mucosal diseases: the inflammatory dermatoses. Aust Dent J. 2010;55:23-38.

24. Yong AA, Tey HL. Paraneoplastic pemphigus. Australas J Dermatol. 2013;54:24150.

25. Wieczorek M, Czernik A. Paraneoplastic pemphigus: a short review. Clin Cosmet Investig Dermatol. 2016;9:291-5.

26. Chan LS. Ocular and oral mucous membrane pemphigoid (cicatricial pemphigoid). Clin Dermatol. 2012;30:34-7.

27. Vorobyev A, Ludwig RJ, Schmidt E. Clinical features and diagnosis of epidermolysis bullosa acquisita. Expert Rev Clin Immunol. 2017;13:157-69.

28. Liang Y, Sarkar MK, Tsoi LC, Gudjonsson JE. Psoriasis: a mixed autoimmune and autoinflammatory disease. Curr Opin Immunol. 2017;49:1-8.
29. Boehncke WH, Schön MP. Psoriasis. Lancet. 2015;386:983-94.

30. Fatahzadeh M, Schwartz RA. Oral psoriasis: an overlooked enigma. Dermatology. 2016;232:319-25.

31. Mattsson U, Warfvinge G, Jontell M. Oral psoriasis-a diagnostic dilemma: a report of two cases and a review of the literature. Oral Surg Oral Med Oral Pathol Oral Radiol. 2015;120:e183-9.

32. Olson MA, Rogers RS 3rd, Bruce AJ. Oral lichen planus. Clin Dermatol. 2016;34: 495-504.

33. Larsen KR, Johansen JD, Reibel J, Zachariae C, Rosing K, Pedersen AML. Oral symptoms and salivary findings in oral lichen planus, oral lichenoid lesions and stomatitis. BMC Oral Health. 2017;17:103.

34. Patil B, Hegde S, Naik S, Sharma R. Oral blistering-report of two cases of erythema multiforme \& literature review. J Clin Diagn Res. 2013;7:2080-3.

35. Kohanim S, Palioura S, Saeed HN, Akpek EK, Amescua G, Basu S, et al. StevensJohnson syndrome / toxic epidermal necrolysis-a comprehensive review and guide to therapy. I. Systemic disease. Ocul Surf. 2016;14:2-19.

36. Sargenti Neto S, de Paulo LF, Rosa RR, Durighetto AF. Stevens-Johnson syndrome: an oral viewpoint. Int J Pediatr Otorhinolaryngol. 2013;77:284-6.

37. Pareira MS, Munerato MC. Oral manifestations of inflammatory bowel diseases: two case reports. Clin Med Res. 2016;14:46-52.

38. Mahvić-Urek M, Tomac-Stojmenović M, Mijandrušić-Sinčić B. Oral pathology in inflammatory bowel disease. World I Gastroenterol. 2016;22:5655-67.

39. Laube R, Liu K, Schifter M, Yang JL, Suen MK, Leong RW. Oral and upper gastrointestinal Crohn's disease. J Gastroenterol Hepatol. 2018;33:355-64.

40. Gajendran M, Loganathan P, Catinella AP, Hashash JG. A comprehensive review and update on Crohn's disease. Dis Mon. 2018;64:20-57.

41. Ślebioda Z, Szponar E, Kowalska A. Etiopathogenesis of recurrent aphthous stomatitis and the role of immunologic aspects: literature review. Arch Immunol Ther Exp. 2014;62:205-15.

42. Chavan M, Jain H, Diwan N, Khedkar S, Shete A, Durkar S. Recurrent aphthous stomatitis: a review. J Oral Pathol Med. 2012;41:577-83.

43. Vivino FB. Sjogren's syndrome: clinical aspects. Clin Immunol. 2017;182:48-54.

44. Gonzales S, Sung H, Sepulveda D, Gonzales MJ, Molina C. Oral manifestations and their treatment in Sjögren's syndrome. Oral Dis. 2014;20:153-61.

45. Cartee DL, Maker S, Dalonges D, Manski MC. Sjögren's syndrome: oral manifestations and treatment, a dental perspective. J Dent Hyg. 2015;89:365-71.

46. Błochowiak K, Olewicz-Gawlik A, Polańska A, Nowak-Gabryel M, Kocięcki J, Witmanowski $\mathrm{H}$, et al. Oral mucosal manifestations in primary and secondary Sjögren syndrome and dry mouth syndrome. Postepy Dermatol Alergol. 2016;33:23-7.

47. Menzies S, O'Shea F, Galvin S, Wynne B. Oral manifestations of lupus. Ir J Med Sci. 2018;187:91-3.

48. Simões DM, Fava M, Figueiredo MA, Salum FG, Cherubini K. Oral manifestations of lupus erythematosus-report of two cases. Gerodontology. 2013;30:303-8.

49. Silvestre-Rangil J, Bagán L, Silvestre FJ, Bagán JV. Oral manifestations of rheumatoid arthritis. A cross-sectional study of 73 patients. Clin Oral Investig. 2016; 20:2575-80.

50. Oldroyd A, Lilleker J, Chinoy H. Idiopathic inflammatory myopathies-a guide to subtypes, diagnostic approach and treatment. Clin Med (Lond). 2017;17:322-8.

51. Clark KEN, Isenberg DA. A review of inflammatory idiopathic myopathy focusing on polymyositis. Eur J Neurol. 2018;25:13-23.

52. Tanaka TI, Geist SM. Dermatomyositis: a contemporary review for oral health care providers. Oral Surg Oral Med Oral Pathol Oral Radiol. 2012;114:e1-8.

53. Fett N. Scleroderma: nomenclature, etiology, pathogenesis, prognosis, and treatments: facts and controversies. Clin Dermatol. 2013;31:432-7.

54. Crincoli V, Fatone L, Fanelli M, Rotolo RP, Chialà A, Favia G, et al. Orofacial manifestations and temporomandibular disorders of systemic scleroderma: an observational study. Int J Mol Sci. 2016;17:1189.

55. Margaix-Muñoz M, Bagán JV, Jiménez Y, Sarrión MG, Poveda-Roda R. Graft-versus-host disease affecting oral cavity. A review. J Clin Exp Dent. 2015;7:e138-45.

56. Regina Torres S. Oral features of graft-versus-host disease. Rev Bras Hematol Hemoter. 2014;36:9-11. 